\title{
Pacific
}

Journal of

Mathematics

\section{CROSSED POINTED CATEGORIES AND THEIR EQUIVARIANTIZATIONS}

\author{
DEEPAK NAIDU
}




\title{
CROSSED POINTED CATEGORIES AND THEIR EQUIVARIANTIZATIONS
}

\author{
DEEPAK NAIDU
}

\begin{abstract}
We propose a notion, quasiabelian third cohomology of crossed modules, which generalizes Eilenberg and Mac Lane's abelian and Ospel's quasiabelian cohomology. We classify crossed pointed categories in terms of it. We apply the process of equivariantization to the latter to obtain braided fusion categories, which may be viewed as generalizations of the categories of modules over twisted Drinfeld doubles of finite groups. As a consequence, we obtain a description of all braided group-theoretical categories. We give a criterion for these categories to be modular. We describe the quasitriangular quasi-Hopf algebras underlying these categories.
\end{abstract}

\section{Introduction}

Turaev's notion [2000; 2008] of a crossed category (short for braided group-crossed category) has attracted much attention recently [Drinfeld et al. 2010; Kirillov 2001a; 2001b; Müger 2004; 2005]. Roughly, a crossed category consists of a group $G$, a $G$-graded tensor category $\mathscr{C}$, an action $g \mapsto T_{g}$ of $G$ on $\mathscr{C}$ by tensor autoequivalences, and $G$-braidings $c(X, Y): X \otimes Y \stackrel{\sim}{\rightarrow} T_{g}(Y) \otimes X$ for $X, Y \in \mathscr{C}$, satisfying certain compatibility conditions. Crossed categories are known to arise in various contexts; for instance, Müger [2004] showed that Galois extensions of braided tensor categories have a natural structure of crossed categories. In [2005], Müger established a connection between 1-dimensional quantum field theories and crossed categories. Kirillov [2001b] showed that crossed categories arise in the theory of vertex operator algebras.

A fusion category is said to be pointed if all its simple objects are invertible. One of the goals of this paper is to classify all crossed pointed categories. From [Joyal and Street 1993], it is known that braided pointed categories are classified by Eilenberg and Mac Lane's abelian cohomology $\mathrm{H}_{\mathrm{ab}}^{3}\left(A, \mathbb{K}^{\times}\right)$, where $A$ is a finite abelian group. On the other hand, certain crossed pointed categories in which the group action is strict were described by Turaev [2000; 2008] in terms of Ospel's quasiabelian cohomology $\mathrm{H}_{\mathrm{qa}}^{3}\left(G, \mathbb{K}^{\times}\right)$, where $G$ is a (not necessarily abelian) finite

MSC2000: primary 18D10; secondary 16W30.

Keywords: braided crossed $G$-categories, equivariantization, group-theoretical fusion categories. 
group. As remarked in [Müger 2005, Section 4.9], to obtain a complete classification of crossed pointed categories one must allow for nonstrict group actions. To this end, Definition 3.4 generalizes Ospel's quasiabelian cohomology to the notion of quasiabelian third cohomology $\mathrm{H}_{\mathrm{qa}}^{3}\left(\mathscr{X}, \mathbb{K}^{\times}\right)$of a crossed module $\mathscr{X}$. To any given $\xi \in Z_{\mathrm{qa}}^{3}\left(\mathscr{X}, \mathbb{K}^{\times}\right)$, we associate a crossed pointed category $\mathscr{C}(\xi)$ and show that all crossed pointed categories are of this form.

Another idea that has been studied extensively recently is that of a modular category. Examples of modular categories arise in quantum group theory, threedimensional topology, vertex operator algebras and rational conformal field theory. Let $G$ be a finite group. Perhaps the most accessible construction of a modular category is that of the category of modules over the Drinfeld double $D(G)$ of $G$. Let $\omega$ be a 3-cocycle on $G$. In [1990; 1992], Dijkgraaf, Pasquier, and Roche introduced a quasitriangular quasi-Hopf algebra $D^{\omega}(G)$, generalizing the Drinfeld double $D(G)$. It is well known that the category $D^{\omega}(G)$-Mod of modules over $D^{\omega}(G)$ is a modular category. Modular categories resembling $D^{\omega}(G)$-Mod arise naturally from crossed pointed categories. An important feature of a general crossed fusion category is that the application of the equivariantization process (which is analogous to taking the invariants under a group action) yields a braided fusion category. We apply the equivariantization process to the crossed pointed category $\mathscr{C}(\xi)$ and study the resulting braided fusion category, which resembles the category $D^{\omega}(G)$-Mod. As a consequence, we obtain a description of all braided group-theoretical categories. In Proposition 5.6, we show that $\mathscr{C}(\xi)$ is modular if and only if $\xi$ is nondegenerate in the sense of Definition 3.10 and a certain homomorphism is surjective.

By a general result, the equivariantization of the category $\mathscr{C}(\xi)$ is equivalent as a braided fusion category to the category of modules over some finite-dimensional quasitriangular quasi-Hopf algebra $H$. In the sequel we describe such an $H$. Namely, given $\xi \in Z_{\text {qa }}^{3}\left(\mathscr{X}, \mathbb{K}^{\times}\right)$, we construct a finite-dimensional quasitriangular quasi-Hopf algebra $H(\xi)$, generalizing $D^{\omega}(G)$, and show that $\mathscr{C}(\xi) \cong H(\xi)$-Mod, as braided fusion categories.

Outline. Section 2 recalls essential definitions and results about nondegenerate fusion categories, equivariantization, and crossed categories. Section 3 proposes the notion of quasiabelian third cohomology of crossed modules. In Section 4, we construct crossed pointed categories from quasiabelian 3-cocycles and classify the former. In Section 5, we apply the process of equivariantization to the categories obtained in Section 4 and study the resulting braided fusion categories. In Section 6, we construct finite-dimensional quasitriangular quasi-Hopf algebras from quasiabelian 3-cocycles and show that these underlie the braided fusion categories obtained in Section 5. 


\section{Preliminaries}

We will freely use the language and basic theory of fusion categories and modular categories [Bakalov and Kirillov 2001; Ostrik 2003; Etingof et al. 2005].

2a. Conventions. Let $\mathbb{K}$ be an algebraically closed field of characteristic 0 . The multiplicative group of nonzero elements of $\mathbb{K}$ will be denoted by $\mathbb{K}^{\times}$. Unless otherwise stated, all cocycles will have coefficients in the trivial module $\mathbb{K}^{\times}$. All functors will be assumed to be additive and $\mathbb{K}$-linear on the morphism spaces. The unit object of a tensor category will be denoted by $\mathbb{1}$. The identity element of a group will be denoted by $e$.

2b. Morita equivalence. Following [Müger 2003a], we say that two fusion categories $\mathscr{C}$ and $\mathscr{D}$ are Morita equivalent if $\mathscr{D}$ is equivalent to the dual fusion category $\mathscr{C}_{\mathcal{M}}^{*}$ for some indecomposable right $\mathscr{C}$-module category $\mathcal{M}$; see also [Etingof et al. 2005; Ospel 1999]. This is known to be an equivalence relation on the class of fusion categories. A fusion category is said to be pointed if all its simple objects are invertible. A fusion category is group-theoretical if it is Morita equivalent to a pointed category.

2c. Nondegenerate fusion categories. Let $\mathscr{C}$ be a braided fusion category with braiding $c$. Following [Müger 2003b], we say two objects $X$ and $Y$ of $\mathscr{C}$ centralize each other if $c(Y, X) \circ c(X, Y)=\mathrm{id}_{X \otimes Y}$.

The centralizer of a fusion subcategory $\mathscr{D} \subseteq \mathscr{C}$ is the full fusion subcategory $\mathscr{D}^{\prime}$ of $\mathscr{C}$ consisting of all objects $X \in \mathscr{C}$ that centralize every object in $\mathscr{D}$. The category $\mathscr{C}$ is said to be nondegenerate if $\mathscr{C}^{\prime}=\mathrm{Vec}$ (the fusion category generated by the unit object). If $\mathscr{C}$ is a premodular category, that is, if it has a twist, then it is nondegenerate if and only if it is modular [Beliakova and Blanchet 2001; Müger 2003b; Drinfeld et al. 2010].

Proposition 2.1. Let $\mathscr{b}$ be a nondegenerate fusion category. Suppose $\mathscr{b}$ admits a twist. Then the set of twists on $\mathscr{b}$ is in bijection with the set of invertible self-dual objects of $\mathscr{C}$.

Proof. Let $\mathrm{Aut}_{\otimes}\left(\mathrm{id}_{\mathscr{C}}\right)$ denote the group of tensor automorphisms of the identity tensor functor $\mathrm{id}_{\mathscr{C}}$. Let $\mathrm{Aut}_{\otimes}^{*}\left(\operatorname{id}_{\mathscr{C}}\right):=\left\{\varphi \in \operatorname{Aut}_{\otimes}\left(\operatorname{id}_{\mathscr{C}}\right) \mid \varphi_{X^{*}}=\left(\varphi_{X}\right)^{*}\right.$ for all $\left.X \in \mathscr{C}\right\}$. Let $\theta$ be a fixed twist on $\mathscr{C}$. The map $\varphi \mapsto \theta_{\varphi}$ defined by $\left(\theta_{\varphi}\right)_{X}:=\theta_{X} \circ \varphi_{X}$ for all $X \in \mathscr{C}$ is a bijection from $\operatorname{Aut}_{\otimes}^{*}\left(\operatorname{id}_{\mathscr{C}}\right)$ to the set of all twists on $\mathscr{C}$.

Let $X_{1}, X_{2}, \ldots$ denote the simple objects of $\mathscr{C}$, and let $G(\mathscr{C})$ denote the group of invertible objects of $\mathscr{C}$. Let $S$ denote the $S$-matrix of $\mathscr{C}$ with respect to $\theta$. It was shown in [Gelaki and Nikshych 2008] that the map

$$
G(\mathscr{C}) \rightarrow \operatorname{Aut}_{\otimes}\left(\operatorname{id}_{\mathscr{C}}\right), \quad X_{j} \mapsto \varphi_{j}, \quad \text { where }\left(\varphi_{j}\right)_{X_{i}}:=\frac{S_{i j}}{d\left(X_{i}\right) d\left(X_{j}\right)} \operatorname{id}_{X_{i}},
$$


is an isomorphism. It is easy to check that this map restricts to a bijection between the set of invertible self-dual objects of $\mathscr{C}$ and the set $\operatorname{Aut}_{\otimes}^{*}\left(\operatorname{id}_{\mathscr{C}}\right)$.

2d. Equivariantization. Recall that a tensor functor between two tensor categories $\mathscr{C}$ and $\mathscr{D}$ is a triple $\left(F, \varphi, \varphi_{0}\right)$, where $F: \mathscr{C} \rightarrow \mathscr{D}$ is a functor, $\varphi$ is a natural isomorphism $F \circ \otimes_{\mathscr{C}} \stackrel{\sim}{\rightarrow} \otimes_{\mathscr{D}} \circ(F \times F)$, and $\varphi_{0}$ is an isomorphism $F\left(\mathbb{1}_{\mathscr{C}}\right) \stackrel{\sim}{\rightarrow} \mathbb{1}_{\mathscr{D}}$ satisfying certain compatibility conditions; see [Kassel 1995]. We will call $\varphi$ the tensor structure on $F$ and $\varphi_{0}$ the unit-preserving structure on $F$. For a group $G$, we will denote by $\underline{G}$ the tensor category whose objects are elements of $G$, whose morphisms are the identities, and whose tensor product is given by the group operation in $G$.

Let $\mathscr{C}$ be a fusion category with an action of a finite group $G$ given by a tensor functor $T: \underline{G} \rightarrow \operatorname{Aut}_{\otimes}(\mathscr{C}), g \mapsto T_{g}$. Let $\gamma$ be the tensor structure on the functor $T$. In this situation one can define a $G$-equivariant object in $\mathscr{C}$ to be a pair $\left(X,\left\{u_{g}\right\}_{g \in G}\right)$ in which $X$ is an object of $\mathscr{C}$ and

$$
u_{g}: T_{g}(X) \stackrel{\sim}{\rightarrow} X \quad \text { for } g \in G,
$$

is a family of isomorphisms, called the equivariant structure on $X$, such that

$$
u_{g h}=u_{g} \circ T_{g}\left(u_{h}\right) \circ \gamma_{g, h}(X) \text { for all } g, h \in G .
$$

One defines morphisms between equivariant objects to be morphisms in $\mathscr{b}$ that commute with the equivariant structures. The equivariantization $\mathscr{C}^{G}$ of $\mathscr{C}$ is the category of $G$-equivariant objects of $\mathscr{C}$ [Kirillov 2001a; Arkhipov and Gaitsgory 2003; Gaitsgory 2005; Tambara 2001]. The equivariantization category $\mathscr{C}^{G}$ is a fusion category with tensor product defined by

$$
\left(X,\left\{u_{g}\right\}_{g \in G}\right) \otimes\left(X^{\prime},\left\{u_{g}^{\prime}\right\}_{g \in G}\right):=\left(X \otimes X^{\prime},\left\{\tilde{u}_{g}\right\}_{g \in G}\right)
$$

for $\left(X,\left\{u_{g}\right\}_{g \in G}\right),\left(X^{\prime},\left\{u_{g}^{\prime}\right\}_{g \in G}\right) \in \mathscr{C}^{G}$, where

$$
\tilde{u}_{g}:=\left(u_{g} \otimes u_{g}^{\prime}\right) \circ \mu_{g}\left(X, X^{\prime}\right)
$$

for all $g \in G$. Here $\mu_{g}$ is the tensor structure on the functor $T_{g}, g \in G$.

Remark 2.2. We have FPdim $\left(\mathscr{C}^{G}\right)=|G| \operatorname{FPdim}(\mathscr{C})$.

2e. Crossed categories. Recall that a grading of a fusion category $\mathscr{C}$ by a finite group $G$ is a decomposition $\mathscr{C}=\bigoplus_{g \in G} \mathscr{C}_{g}$ of $\mathscr{C}$ into a direct sum of full abelian subcategories such that $\otimes$ maps $\mathscr{C}_{g} \times \mathscr{C}_{h}$ to $\mathscr{C}_{g h}$ and $*$ maps $\mathscr{C}_{g}$ to $\mathscr{C}_{g^{-1}}$ for all $g, h \in G$. Note that $\mathscr{C}_{e}$, called the trivial component, is a fusion subcategory of $\mathscr{C}$. A grading is said to faithful if $\mathscr{b}_{g} \neq 0$ for all $g \in G$.

Below we recall the notion of a crossed category (short for braided groupcrossed category), introduced by Turaev [2000; 2008] in a more general form; see also [Drinfeld et al. 2010; Müger 2004; 2005]. 
Definition 2.3. A crossed fusion category is an octuple $(\mathscr{C}, G, T, \gamma, l, \mu, v, c)$ in which

- $G$ is a finite group;

- $\mathscr{C}$ is a fusion category with (not necessarily faithful) $G$-grading $\mathscr{C}=\bigoplus_{g \in G} \mathscr{b}_{g}$;

- $T: \underline{G} \rightarrow \operatorname{Aut}_{\otimes}(\mathscr{C}), g \mapsto T_{g}$ is a tensor functor satisfying $T_{g}\left(\mathscr{C}_{h}\right) \subset \mathscr{C}_{g h g^{-1}}$, with tensor structure $\gamma$ and unit-preserving structure $l$;

- $\mu$ is a family $\left\{\mu_{g}\right\}_{g \in G}$, where $\mu_{g}$ is a tensor structure on $T_{g}$;

- $v$ is a family $\left\{v_{g}\right\}_{g \in G}$, where $v_{g}$ is a unit-preserving structure on $T_{g}$;

- $c(X, Y): X \otimes Y \stackrel{\sim}{\rightarrow} T_{g}(Y) \otimes X$ for $X \in \mathscr{C}_{g}$ and $Y \in \mathscr{C}$ is a family of natural isomorphisms, called $G$-braiding;

and the following compatibility conditions are satisfed:

(i) $\quad\left(\gamma_{g, h}(Y) \otimes \operatorname{id}_{T_{g}(X)}\right) \circ\left(\gamma_{g h g^{-1}, g}^{-1}(Y) \otimes \operatorname{id}_{T_{g}(X)}\right) \circ c\left(T_{g}(X), T_{g}(Y)\right) \circ \mu_{g}(X, Y)$

$$
=\mu_{g}\left(T_{h}(Y), X\right) \circ T_{g}(c(X, Y))
$$

for all $g, h \in G$ and objects $X \in \mathscr{C}_{h}$ and $Y \in \mathscr{C}$.

$$
\begin{aligned}
\alpha_{T_{g}\left(T_{h}(Z)\right), X, Y}^{-1} \circ\left(\gamma_{g, h}(Z)\right. & \left.\otimes \operatorname{id}_{X \otimes Y}\right) \circ c(X \otimes Y, Z) \circ \alpha_{X, Y, Z}^{-1} \\
= & \left(c\left(X, T_{h}(Z)\right) \otimes \operatorname{id}_{Y}\right) \circ \alpha_{X, T_{h}(Z), Y}^{-1} \circ\left(\operatorname{id}_{X} \otimes c(Y, Z)\right),
\end{aligned}
$$

for all $g, h \in G$ and objects $X \in \mathscr{C}_{g}, Y \in \mathscr{C}_{h}$ and $Z \in \mathscr{C}$.

$$
\begin{aligned}
\alpha_{T_{g}(Y), T_{g}(Z), X} \circ\left(\mu_{g}(Y, Z) \otimes \operatorname{id}_{X}\right) \circ c(X, Y \otimes Z) \circ \alpha_{X, Y, Z} \\
=\left(\operatorname{id}_{T_{g}(Y)} \otimes c(X, Z)\right) \circ \alpha_{T_{g}(Y), X, Z} \circ\left(c(X, Y) \otimes \operatorname{id}_{Z}\right),
\end{aligned}
$$

for all $g \in G$ and objects $X \in \mathscr{C}_{g}$ and $Y, Z \in \mathscr{C}$.

(Here $\alpha$ denotes the associativity constraint of $\mathscr{C}$.)

Remark 2.4. The trivial component of a crossed fusion category is a braided fusion category.

Now let $\mathscr{C}:=(\mathscr{C}, G, T, \gamma, l, \mu, v, c)$ be a crossed fusion category. Kirillov [2001a] and Müger [2004] explain that the equivariantization category $\mathscr{C}^{G}$ admits a braiding, that is, $\mathscr{C}^{G}$ is a braided fusion category. The braiding $\tilde{c}$ on $\mathscr{C}^{G}$ is defined as follows. Let $\left(X,\left\{u_{g}\right\}_{g \in G}\right)$ and $\left(X^{\prime},\left\{u_{g}^{\prime}\right\}_{g \in G}\right)$ be objects of $\mathscr{C}^{G}$. Let $X=\bigoplus_{g \in G} X_{g}$ be a decomposition of $X$ with respect to the $G$-grading of $\mathscr{C}$. Then $\tilde{c}_{X, X^{\prime}}$ is given by the composition

(4) $\quad X \otimes X^{\prime}=\bigoplus_{g \in G} X_{g} \otimes X^{\prime} \stackrel{\oplus c_{X_{g}, X^{\prime}}}{\longrightarrow} \bigoplus_{g \in G} T_{g}\left(X^{\prime}\right) \otimes X_{g}$

$$
\stackrel{\oplus u_{g}^{\prime} \otimes \mathrm{id}_{X_{g}}}{\longrightarrow} \bigoplus_{g \in G} X^{\prime} \otimes X_{g}=X^{\prime} \otimes X .
$$


Remark 2.5. It is shown in [Drinfeld et al. 2010] that the equivariantization category $\mathscr{C}^{G}$ is nondegenerate if and only if the $G$-grading is faithful and the trivial component $\mathscr{C}_{e}$ is nondegenerate.

Definition 2.6. Consider two crossed fusion categories $\mathscr{C}=(\mathscr{C}, G, T, \gamma, \imath, \mu, v, c)$ and $\mathscr{C}^{\prime}=\left(\mathscr{C}^{\prime}, G^{\prime}, T^{\prime}, \gamma^{\prime}, \imath^{\prime}, \mu^{\prime}, v^{\prime}, c^{\prime}\right)$. A crossed tensor functor from $\mathscr{C}$ to $\mathscr{C}^{\prime}$ is a quintuple $\left(f, F, \eta, \eta_{0}, \beta\right)$ in which

- $f: G \rightarrow G^{\prime}$ is a group homomorphism,

- $F: \mathscr{C} \rightarrow \mathscr{C}^{\prime}$ is a tensor functor with tensor structure $\eta$ and unit-preserving structure $\eta_{0}$, and

- $\beta$ is a family $\left\{\beta_{g}\right\}_{g \in G}$, where $\beta_{g}: F \circ T_{g} \stackrel{\sim}{\rightarrow} T_{f(g)}^{\prime} \circ F$ is an isomorphism of tensor functors,

and the following compatibility conditions are satisfed:

(i) $F\left(\mathscr{C}_{g}\right) \subseteq \mathscr{C}_{f(g)}^{\prime}$ for all $g \in G$.

(ii) $\left(\beta_{g}(Y) \otimes \operatorname{id}_{F(X)}\right) \circ \eta\left(T_{g}(Y), X\right) \circ F(c(X, Y))=c^{\prime}(F(X), F(Y)) \circ \eta(X, Y)$ for all $g \in G$ and objects $X \in \mathscr{C}_{g}$ and $Y \in \mathscr{C}$.

(iii) $T_{f(g)}^{\prime}\left(\beta_{h}(X)\right) \circ \beta_{g}\left(T_{h}(X)\right) \circ F\left(\gamma_{g, h}(X)\right)=\gamma_{f(g), f(h)}^{\prime}(F(X)) \circ \beta_{g h}(X)$ for all $g, h \in G$ and objects $X \in \mathscr{C}$.

We say that $\left(f, F, \eta, \eta_{0}, \beta\right)$ is an equivalence if $f$ is an isomorphism and $F$ is an equivalence.

2f. Pointed categories. A fusion category is said to be pointed if all its simple objects are invertible.

Let $X$ be a finite group and $\omega$ be a 3-cocycle on $X$. We associate to the pair $(X, \omega)$ a pointed category $\operatorname{Vec}_{X}^{\omega}$ whose objects are $X$-graded finite-dimensional vector spaces over $\mathbb{K}$, whose morphisms are linear transformations that respect the grading, and whose unit object is the ground field $\mathbb{K}$ supported on $\{e\}$. The tensor product $V \otimes W$ of homogeneous objects $V, W \in \operatorname{Vec}_{X}^{\omega}$ of degrees $x, y \in X$, respectively, is defined to be the homogeneous object $V \otimes_{\mathbb{K}} W$ of degree $x y$.

The associativity constraint $\alpha$ is defined by $\alpha_{U, V, W}:(U \otimes V) \otimes W \stackrel{\sim}{\rightarrow} U \otimes(V \otimes W), \quad(u \otimes v) \otimes w \mapsto \omega(x, y, z) u \otimes(v \otimes w)$, where $U, V, W \in \operatorname{Vec}_{G}^{\omega}$ and $u \in U, v \in V, w \in W$ are homogeneous elements of degrees $x, y, z \in X$, respectively.

The left and right unit constraints $\lambda$ and $\rho$, respectively, are defined by

$$
\begin{aligned}
& \lambda_{V}:=\mathbb{K} \otimes V \stackrel{\sim}{\rightarrow} V, \quad 1 \otimes v \mapsto \omega(e, e, x)^{-1} v, \\
& \rho_{V}:=V \otimes \mathbb{K} \stackrel{\sim}{\rightarrow} V, \quad v \otimes 1 \mapsto \omega(x, e, e) v,
\end{aligned}
$$

where $V \in \operatorname{Vec}_{G}^{\omega}$ and $v \in V$ is a homogeneous element of degree $x \in X$. 
Every pointed category is equivalent to some $\operatorname{Vec}_{X}^{\omega}$.

2g. Crossed modules. Recall that a (finite) crossed module is a triple $(G, X, \partial)$, where $G$ and $X$ are (finite) groups with $G$ acting on $X$ as automorphisms, denoted $(g, x) \mapsto{ }^{g} x$, and $\partial: X \rightarrow G$ is a homomorphism satisfying

$$
\begin{array}{ll}
\partial(x) x^{\prime}=x x^{\prime} x^{-1} & \text { for } x, x^{\prime} \in X, \\
\partial\left({ }^{g} x\right)=g \partial(x) g^{-1} & \text { for } g \in G \text { and } x \in X .
\end{array}
$$

Note that Ker $\partial$ is a central subgroup of $X$.

A homomorphism of crossed modules $(G, X, \partial) \rightarrow\left(G^{\prime}, X^{\prime}, \partial^{\prime}\right)$ is a pair of group homomorphisms $\left(f: G \rightarrow G^{\prime}, F: X \rightarrow X^{\prime}\right)$ such that $\partial^{\prime} \circ F=f \circ \partial$ and $F\left({ }^{g} x\right)={ }^{f(g)} F(x)$ for $g \in G$. We say that $(f, F)$ is an isomorphism if both $f$ and $F$ are isomorphisms.

\section{Quasiabelian third cohomology of crossed modules}

Let $A$ be an abelian group. Eilenberg and Mac Lane [Eilenberg and Mac Lane 1953; 1954; Mac Lane 1952] argue that the cohomology groups $H^{n}\left(A, \mathbb{K}^{\times}\right)$are inappropriate since they do not take into account the abelianness of $A$, and so should be replaced by groups $\mathrm{H}_{\mathrm{ab}}^{n}\left(A, \mathbb{K}^{\times}\right)$. (For the cohomology theory for crossed modules, see [Whitehead 1949].) Below we recall the definition of $\mathrm{H}_{\mathrm{ab}}^{3}\left(A, \mathbb{K}^{\times}\right)$.

An abelian 3-cocycle on $A$ is a pair $(\omega, c)$, where $\omega$ is a normalized 3-cocycle on $A$, that is, for all $w, x, y, z \in A$,

$$
\begin{aligned}
& \omega(x, y, z)=1 \text { if } x, y \text { or } z \text { is the identity, } \\
& \omega(x, y, z) \omega(w, x y, z) \omega(w, x, y)=\omega(w, x, y z) \omega(w x, y, z)
\end{aligned}
$$

and $c$ is a 2-cochain on $A$ (that is, $c \in C^{2}\left(A, \mathbb{K}^{\times}\right)$) satisfying the equations

$$
\begin{aligned}
& c(x y, z)=\frac{\omega(x, y, z) \omega(z, x, y)}{\omega(x, z, y)} c(x, z) c(y, z), \\
& c(x, y z)=\frac{\omega(y, x, z)}{\omega(x, y, z) \omega(y, z, x)} c(x, y) c(x, z)
\end{aligned}
$$

for all $x, y, z \in A$.

Abelian 3-cocycles on $A$ form an abelian group, denoted by $Z_{\mathrm{ab}}^{3}\left(A, \mathbb{K}^{\times}\right)$, under pointwise multiplication. The group of coboundaries is defined by

$$
B_{\mathrm{ab}}^{3}\left(A, \mathbb{K}^{\times}\right):=\left\{\left(d \eta,(x, y) \mapsto \frac{\eta(y, x)}{\eta(x, y)}\right) \mid \text { normalized } \eta \in C^{2}\left(G, \mathbb{K}^{\times}\right)\right\},
$$

which is a subgroup of $Z_{\mathrm{ab}}^{3}\left(A, \mathbb{K}^{\times}\right)$. The quotient $Z_{\mathrm{ab}}^{3}\left(A, \mathbb{K}^{\times}\right) / B_{\mathrm{ab}}^{3}\left(A, \mathbb{K}^{\times}\right)$is the abelian third cohomology of $A$, denoted $\mathrm{H}_{\mathrm{ab}}^{3}\left(A, \mathbb{K}^{\times}\right)$. 
Remark 3.1. The group $\mathrm{H}_{\mathrm{ab}}^{3}\left(A, \mathbb{K}^{\times}\right)$is isomorphic to the group of quadratic forms on $A$; see [Mac Lane 1952].

Definition 3.2. We say an abelian 3-cocycle $(\omega, c)$ on $A$ is nondegenerate if the symmetric bicharacter $A \times A \rightarrow \mathbb{K}^{\times},(x, y) \mapsto c(y, x) c(x, y)$ is nondegenerate.

In [1999], C. Ospel generalized the notion of abelian third cohomology in the following way. Let $G$ be a (not necessarily abelian) group. A quasiabelian 3cocycle on $G$ is a pair $(\omega, c)$, where $\omega$ is a 3-cocycle on $G$ and $c$ is a 2-cochain on $G$ (that is, $c \in C^{2}\left(G, \mathbb{K}^{\times}\right)$) satisfying for all $g, x, y, z \in G$ the equations

$$
\begin{aligned}
\omega\left(g x g^{-1}, g y g^{-1}, g z g^{-1}\right) & =\omega(x, y, z), \\
c\left(g x g^{-1}, g y g^{-1}\right) & =c(x, y), \\
c(x y, z) & =\frac{\omega(x, y, z) \omega\left(x y z(x y)^{-1}, x, y\right)}{\omega\left(x, y z y^{-1}, y\right)} c\left(x, y z y^{-1}\right) c(y, z), \\
c(x, y z) & =\frac{\omega\left(x y x^{-1}, x, z\right)}{\omega(x, y, z) \omega(y, z, x)} c(x, y) c(x, z),
\end{aligned}
$$

Note 3.3. The third equation above appeared in a slightly different but equivalent form in [Ospel 1999].

Quasiabelian 3-cocycles on $G$ form an abelian group, denoted by $Z_{\mathrm{qa}}^{3}\left(G, \mathbb{K}^{\times}\right)$, under pointwise multiplication. The group of coboundaries is defined by

$$
\begin{aligned}
& B_{\mathrm{qa}}^{3}\left(G, \mathbb{K}^{\times}\right) \\
&:=\left\{\left(d(\eta),(x, y) \mapsto \frac{\eta(y, x)}{\eta(x, y)}\right) \mid \text { conjugation-invariant } \eta \in C^{2}\left(G, \mathbb{K}^{\times}\right)\right\},
\end{aligned}
$$

which is a subgroup of $Z_{\mathrm{qa}}^{3}\left(G, \mathbb{K}^{\times}\right)$. The quotient $Z_{\mathrm{qa}}^{3}\left(G, \mathbb{K}^{\times}\right) / B_{\mathrm{qa}}^{3}\left(G, \mathbb{K}^{\times}\right)$is the quasiabelian third cohomology of $G$, denoted $\mathrm{H}_{\mathrm{qa}}^{3}\left(G, \mathbb{K}^{\times}\right)$. If $G$ is abelian, quasiabelian cohomology reduces to abelian cohomology: $\mathrm{H}_{\mathrm{ab}}^{3}\left(G, \mathbb{K}^{\times}\right)=\mathrm{H}_{\mathrm{qa}}^{3}\left(G, \mathbb{K}^{\times}\right)$.

We can extend Ospel's quasiabelian cohomology for groups to cover crossed modules: We allow $G$ to act on an arbitrary group $X$ (not just $X=G$ ). The first condition, $\omega^{g}=\omega$, in Ospel's definition is replaced by the condition that $\omega^{g}$ is cohomologous to $\omega$ via $\mu_{g}$. The second condition, $c^{g}=c$, is extended similarly, as are the others. This results in the following definition, whose main motivation is the classification of crossed pointed categories (see Section 4).

Definition 3.4. A quasiabelian 3-cocycle on a crossed module $\mathscr{X}=(G, X, \partial)$ is a quadruple $(\omega, \gamma, \mu, c)$, where

(a) $\omega \in Z^{3}\left(X, \mathbb{K}^{\times}\right)$,

(b) $\gamma \in Z^{2}\left(G, C^{1}\left(X, \mathbb{K}^{\times}\right)\right)$, 
(c) $\mu \in C^{1}\left(G, C^{2}\left(X, \mathbb{K}^{\times}\right)\right)$and satisfies $d\left(\mu_{g}\right)=\omega^{g} / \omega$ for $g \in G$, that is,

$$
\frac{\mu_{g}(y, z) \mu_{g}(x, y z)}{\mu_{g}(x y, z) \mu_{g}(x, y)}=\frac{\omega^{g}(x, y, z)}{\omega(x, y, z)} \quad \text { for } g \in G \text { and } x, y, z \in X,
$$

(d) $d\left(\gamma_{g, h}\right)=(d \mu)_{g, h}$ for $g, h \in G$, that is,

$$
\frac{\gamma_{g, h}(x) \gamma_{g, h}(y)}{\gamma_{g, h}(x y)}=\frac{\mu_{g}\left({ }^{h} x,{ }^{h} y\right) \mu_{h}(x, y)}{\mu_{g h}(x, y)} \text { for } g, h \in G \text { and } x, y \in X,
$$

(e) $c \in C^{2}\left(X, \mathbb{K}^{\times}\right)$and satisfies

(e $\left.\mathrm{e}_{1}\right) \frac{c^{g}(x, y)}{c(x, y)}=\frac{\mu_{g}\left(x y x^{-1}, x\right)}{\mu_{g}(x, y)} \frac{\gamma_{g \partial(x) g^{-1}, g}(y)}{\gamma_{g, \partial(x)}(y)} \quad$ for $g \in G, x, y \in X$,

( $\left.\mathrm{e}_{2}\right) \quad c(x y, z)=\frac{\omega(x, y, z) \omega\left((x y) z(x y)^{-1}, x, y\right)}{\omega\left(x, y z y^{-1}, y\right) \gamma_{\partial(x), \partial(y)}(z)} c\left(x, y z y^{-1}\right) c(y, z)$ for $x, y, z \in X$,

(e $\left.\mathrm{e}_{3}\right) \quad c(x, y z)=\frac{\omega\left(x y x^{-1}, x, z\right)}{\omega(x, y, z) \omega\left(x y x^{-1}, x z x^{-1}, x\right) \mu_{\partial(x)}(y, z)} c(x, y) c(x, z)$ for $x, y, z \in X$.

Note 3.5. Here $C^{n}$ denotes the space of $n$-cochains, $Z^{n}$ denotes the space of $n$ cocycles, and $d$ is the usual differential operator [Brown 1982]. (The definition of $d$ depends on whether the module under consideration is left or right.) The action $(g, x) \mapsto{ }^{g} X$ of $G$ on $X$ induces a right action of $G$ on $C^{n}\left(X, \mathbb{K}^{\times}\right)$by translations. The map $c^{g} \in C^{2}\left(X, \mathbb{K}^{\times}\right)$is defined by $c^{g}(x, y):=c\left({ }^{g} x,{ }^{g} y\right)$ and the map $\omega^{g}$ is defined similarly.

Quasiabelian 3-cocycles on a crossed module $\mathscr{X}=(G, X, \partial)$ form an abelian group $Z_{\mathrm{qa}}^{3}\left(\mathscr{X}, \mathbb{K}^{\times}\right)$under pointwise multiplication.

We define the group of coboundaries by

$$
\begin{aligned}
B_{\mathrm{qa}}^{3}\left(\mathscr{X}, \mathbb{K}^{\times}\right):=\left\{\left(d \eta, d \beta, g \mapsto d\left(\beta_{g}\right) \frac{\eta^{g}}{\eta},(x, y) \mapsto \beta_{\partial(x)}(y) \frac{\eta\left(x y x^{-1}, x\right)}{\eta(x, y)}\right)\right. & \begin{array}{l}
\eta \in C^{2}\left(X, \mathbb{K}^{\times}\right), \\
\beta \in C^{1}\left(G, C^{1}\left(X, \mathbb{K}^{\times}\right)\right)
\end{array}
\end{aligned} .
$$

A direct computation shows that $B_{\mathrm{qa}}^{3}\left(\mathscr{X}, \mathbb{K}^{\times}\right) \subseteq Z_{\mathrm{qa}}^{3}\left(\mathscr{X}, \mathbb{K}^{\times}\right)$.

Definition 3.6. The quasiabelian third cohomology of a crossed module $\mathscr{X}$ is the quotient of $Z_{\mathrm{qa}}^{3}\left(\mathscr{X}, \mathbb{K}^{\times}\right)$by $B_{\mathrm{qa}}^{3}\left(\mathscr{X}, \mathbb{K}^{\times}\right)$. We denote it by $\mathrm{H}_{\mathrm{qa}}^{3}\left(\mathscr{X}, \mathbb{K}^{\times}\right)$.

Remark 3.7. Let $G$ be a group. Consider the crossed module $\mathscr{G}=\left(G, G, \operatorname{id}_{G}\right)$, where $G$ acts on itself by conjugation. 
(i) There is a homomorphism $\mathrm{H}_{\mathrm{qa}}^{3}\left(G, \mathbb{K}^{\times}\right) \rightarrow \mathrm{H}_{\mathrm{qa}}^{3}\left(\mathscr{G}, \mathbb{K}^{\times}\right)$induced from

$$
Z_{\mathrm{qa}}^{3}\left(G, \mathbb{K}^{\times}\right) \rightarrow Z_{\mathrm{qa}}^{3}\left(\mathscr{G}, \mathbb{K}^{\times}\right), \quad(\omega, c) \mapsto(\omega, 1,1, c) .
$$

(ii) There exists a homomorphism $\mathrm{H}^{3}\left(G, \mathbb{K}^{\times}\right) \rightarrow \mathrm{H}_{\mathrm{qa}}^{3}\left(\mathscr{G}, \mathbb{K}^{\times}\right)$; see Lemma 6.3.

Definition 3.8. A quasiabelian 3-cocycle $(\omega, \gamma, \mu, c)$ is normalized if $\omega(x, y, z)=1$ if $x, y$ or $z$ is the identity, $\gamma_{g, h}(x)=1$ if $g, h$ or $x$ is the identity, $\mu_{g}(x, y)=1$ if $x, y$ or $g$ is the identity, $c(x, y)=1$ if $x$ or $y$ is the identity.

Note 3.9. Every quasiabelian 3-cocycle is cohomologous to a normalized one.

Let $(\omega, \gamma, \mu, c)$ be a normalized quasiabelian 3-cocycle on a crossed module $(G, X, \partial)$. Then $\left(\left.\omega\right|_{\operatorname{Ker} \partial},\left.c\right|_{\operatorname{Ker} \partial}\right)$ is an abelian 3-cocycle on the (abelian) group Ker $\partial$.

Definition 3.10. A normalized quasiabelian 3-cocycle $(\omega, \gamma, \mu, c)$ on a crossed module $(G, X, \partial)$ is nondegenerate if the abelian 3-cocycle $\left(\left.\omega\right|_{\operatorname{Ker} \partial},\left.c\right|_{\operatorname{Ker} \partial}\right)$ on the (abelian) group Ker $\partial$ is nondegenerate.

Any homomorphism $(f, F):\left(G^{\prime}, X^{\prime}, \partial^{\prime}\right)=\mathscr{X}^{\prime} \rightarrow \mathscr{X}=(G, X, \partial)$ of crossed modules induces a homomorphism

$$
Z_{\mathrm{qa}}^{3}\left(\mathscr{X}, \mathbb{K}^{\times}\right) \rightarrow Z_{\mathrm{qa}}^{3}\left(\mathscr{L}^{\prime}, \mathbb{K}^{\times}\right), \quad(\omega, \gamma, \mu, c) \mapsto(\omega, \gamma, \mu, c)^{(f, F)},
$$

where

$(\omega, \gamma, \mu, c)^{(f, F)}=\left(\omega \circ F^{\times 3},(g, h) \mapsto \gamma_{f(g), f(h)} \circ F, g \mapsto \mu_{f(g)} \circ F^{\times 2}, c \circ F^{\times 2}\right)$.

It is straightforward to check that this homomorphism preserves coboundaries and thereby provides a homomorphism $\mathrm{H}_{\mathrm{qa}}^{3}\left(\mathscr{X}, \mathbb{K}^{\times}\right) \rightarrow \mathrm{H}_{\mathrm{qa}}^{3}\left(\mathscr{X}^{\prime}, \mathbb{K}^{\times}\right)$. Consequently, for any crossed module $\mathscr{L}$ there is a natural action of the group of automorphisms $\operatorname{Aut}(\mathscr{X})$ of $\mathscr{X}$ on $\mathrm{H}_{\mathrm{qa}}^{3}\left(\mathscr{X}, \mathbb{K}^{\times}\right)$.

\section{Classification of crossed pointed categories}

In this section we classify crossed pointed categories in terms of quasiabelian third cohomology of crossed modules.

4a. Construction of a crossed pointed category from a quasiabelian 3-cocyle on a crossed module. To a quasiabelian 3-cocycle $(\omega, \gamma, \mu, c)$ on a finite crossed module $(G, X, \partial)$, we associate a crossed pointed category $(\mathscr{C}, G, T, \tilde{\gamma}, \imath, \tilde{\mu}, \nu, \tilde{c})$ as follows. As a fusion category, $\mathscr{C}=\operatorname{Vec}_{X}^{\omega}$. For each $g \in G$, let $\mathscr{b}_{g}$ denote the full abelian subcategory consisting of objects of $\operatorname{Vec}_{X}^{\omega}$ supported on $\partial^{-1}(g) \subset X$, that is, objects of $\mathscr{C}_{g}$ are defined to be finite-dimensional $\partial^{-1}(g)$-graded vector spaces (we set $\mathscr{C}_{g}:=\{0\}$ if $\partial^{-1}(g)$ is empty). This defines a $G$-grading $\mathscr{C}=\bigoplus_{g \in G} \mathscr{C}_{g}$ of $\mathscr{C}$. 
Next we define a functor $T: \underline{G} \rightarrow \operatorname{Aut}_{\otimes}(\mathscr{C}), g \mapsto T_{g}$ as follows. Let $V \in \operatorname{Vec}_{X}^{\omega}$ be a homogeneous object of degree $x \in X$. The functor $T_{g}: \operatorname{Vec}_{X}^{\omega} \stackrel{\sim}{\rightarrow} \operatorname{Vec}_{X}^{\omega}$ is defined by $T_{g}(V):=V$ (as a vector space) and the degree of $T_{g}(V)$ is defined to be ${ }^{g} x$. The $T_{g}$ are extended to nonhomogeneous objects and morphisms in the obvious way.

The tensor structure $\tilde{\gamma}$ on the functor $T: \underline{G} \rightarrow \operatorname{Aut}_{\otimes}(\mathscr{C})$ is defined by

$$
\gamma_{g, h}(x) \operatorname{id}_{V}=: \tilde{\gamma}_{g, h}(V): T_{g h}(V) \stackrel{\sim}{\rightarrow}\left(T_{g} \circ T_{h}\right)(V)
$$

for all homogeneous objects $V \in \operatorname{Vec}_{X}^{\omega}$ of degree $x \in X$, and $g, h \in G$.

The unit-preserving structure $l: T_{e} \stackrel{\sim}{\rightarrow} \mathrm{id}_{\mathscr{C}}$ on the functor $T: \underline{G} \rightarrow \operatorname{Aut}_{\otimes}(\mathscr{C})$ is defined by

$$
\gamma_{e, e}^{-1}(x) \operatorname{id}_{V}=: \iota(V): T_{e}(V) \stackrel{\sim}{\rightarrow} \operatorname{id}_{\mathscr{C}}(V)
$$

for all homogeneous objects $V \in \operatorname{Vec}_{X}^{\omega}$ of degree $x \in X$.

The tensor structure $\tilde{\mu}_{g}$ on the functor $T_{g}: \operatorname{Vec}_{X}^{\omega} \stackrel{\sim}{\rightarrow} \operatorname{Vec}_{X}^{\omega}$ for $g \in G$ is defined by

$$
\mu_{g}(x, y) \operatorname{id}_{V \otimes \mathbb{K} W}=: \tilde{\mu}_{g}(V, W): T_{g}(V \otimes W) \stackrel{\sim}{\rightarrow} T_{g}(V) \otimes T_{g}(W)
$$

for all homogeneous objects $V, W \in \operatorname{Vec}_{X}^{\omega}$ of degrees $x, y \in X$, respectively.

The unit-preserving structure $v_{g}$ on the functor $T_{g}: \operatorname{Vec}_{X}^{\omega} \stackrel{\sim}{\rightarrow} \operatorname{Vec}_{X}^{\omega}$ for $g \in G$ is defined by

$$
\mu_{g}^{-1}(e, e) \operatorname{id}_{\mathbb{K}}=: v_{g}: T_{g}(\mathbb{K}) \stackrel{\sim}{\rightarrow} \mathbb{K} .
$$

For $V, W \in$ Vec, let $\tau_{V, W}$ denote the flip operator $V \otimes_{\mathbb{K}} W \stackrel{\sim}{\rightarrow} W \otimes_{\mathbb{K}} V$ that takes $v \otimes_{\mathbb{K}} w \mapsto w \bigotimes_{\mathbb{K}} v$. The $G$-braiding $\tilde{c}$ is defined by

$$
c(x, y) \tau_{V, W}=: \tilde{c}(V, W): V \otimes W \stackrel{\sim}{\rightarrow} T_{g}(W) \otimes V
$$

for all homogeneous objects $V, W \in \operatorname{Vec}_{X}^{\omega}$ of degrees $x, y \in X$. Here $g=\partial(x)$.

The crossed module axioms of $(G, X, \partial)$ and the quasiabelian 3-cocycle axioms of $(\omega, \gamma, \mu, c)$ together ensure that the necessary axioms of a crossed category are satisfied. Specifically, Definition 3.4(c) ensures that $\tilde{\mu_{g}}$ is a tensor structure on the functor $T_{g}$ defined above. Definition 3.4(d) of ensures that $\tilde{\gamma}$ is a tensor structure on the functor $T$. The conditions of Definition 3.4 $\left(\mathrm{e}_{1}\right)-\left(\mathrm{e}_{3}\right)$ correspond to the axioms of Definition 3.4(i)-(iii), respectively.

We will denote the crossed pointed category constructed above by $\mathscr{C}(\omega, \gamma, \mu, c)$.

Remark 4.1. The trivial component $\mathscr{C}(\omega, \gamma, \mu, c)_{e}$ of $\mathscr{C}(\omega, \gamma, \mu, c)$ (under the $G$-grading) is a braided fusion category. As a fusion category, $\mathscr{C}(\omega, \gamma, \mu, c)_{e}=$ $\operatorname{Vec}_{\operatorname{Ker} \partial}^{\left.\omega\right|_{\text {Ker }} \partial}$. Suppose that the quasiabelian 3-cocycle $(\omega, \gamma, \mu, c)$ is normalized. Then the braiding on the trivial component is given by

$$
V \otimes W \rightarrow W \otimes V, \quad v \otimes w \mapsto c(x, y) w \otimes v
$$


for all homogeneous objects $V, W \in \operatorname{Vec}_{\operatorname{Ker} \partial}^{\omega \mid \operatorname{Ker} \partial}$ of degrees $x, y \in \operatorname{Ker} \partial$. Clearly, the braided fusion category $\mathscr{C}(\omega, \gamma, \mu, c)_{e}$ is nondegenerate if and only if the quasiabelian 3-cocycle $(\omega, \gamma, \mu, c)$ is nondegenerate in the sense of Definition 3.10.

\section{4b. Classification.}

Proposition 4.2. Let $\mathscr{C}(\omega, \gamma, \mu, c)$ and $\mathscr{C}\left(\omega^{\prime}, \gamma^{\prime}, \mu^{\prime}, c^{\prime}\right)$ be crossed pointed categories as constructed in Section $4 a$. Then $\mathscr{C}(\omega, \gamma, \mu, c) \cong \mathscr{C}\left(\omega^{\prime}, \gamma^{\prime}, \mu^{\prime}, c^{\prime}\right)$ as crossed categories if and only if there is an isomorphism $(f, F)$ of the underlying (finite) crossed modules such that the quasiabelian 3-cocycles $\left(\omega^{\prime}, \gamma^{\prime}, \mu^{\prime}, c^{\prime}\right)^{(f, F)}$ and $(\omega, \gamma, \mu, c)$ are cohomologous.

Proof. Suppose $(G, X, \partial)$ and $\left(G^{\prime}, X^{\prime}, \partial^{\prime}\right)$ are the underlying (finite) crossed modules of $\mathscr{C}(\omega, \gamma, \mu, c)$ and $\mathscr{C}\left(\omega^{\prime}, \gamma^{\prime}, \mu^{\prime}, c^{\prime}\right)$, respectively. Let $(f, F)$ be an isomorphism from $(G, X, \partial)$ to $\left(G^{\prime}, X^{\prime}, \partial^{\prime}\right)$ such that the quasiabelian 3-cocycles $\left(\omega^{\prime}, \gamma^{\prime}, \mu^{\prime}, c^{\prime}\right)^{(f, F)}$ and $(\omega, \gamma, \mu, c)$ are cohomologous via $(\eta, \beta)$; see Section 3. In what follows we will construct an equivalence $\left(f, \tilde{F}, \tilde{\eta}, \eta_{0}, \tilde{\beta}\right)$ of crossed categories (see Definition 2.6) from $\mathscr{C}(\omega, \gamma, \mu, c)$ to $\mathscr{C}\left(\omega^{\prime}, \gamma^{\prime}, \mu^{\prime}, c^{\prime}\right)$.

Recall that $\mathscr{C}(\omega, \gamma, \mu, c)=\operatorname{Vec}_{X}^{\omega}$ and $\mathscr{C}\left(\omega^{\prime}, \gamma^{\prime}, \mu^{\prime}, c^{\prime}\right)=\operatorname{Vec}_{X^{\prime}}^{\omega^{\prime}}$ as fusion categories. Let $V \in \operatorname{Vec}_{X}^{\omega}$ be a homogeneous object of degree $x \in X$. Define a functor $\tilde{F}: \operatorname{Vec}_{X}^{\omega} \rightarrow \operatorname{Vec}_{X^{\prime}}^{\omega^{\prime}}$ by $\tilde{F}(V):=V$ (as a vector space), and define the degree of $\tilde{F}(V)$ to be $F(x)$. The functor $\tilde{F}$ extends to nonhomogeneous objects and morphisms in the obvious way.

The tensor structure $\tilde{\eta}$ on the functor $\tilde{F}$ is defined by

$$
\eta(x, y) \operatorname{id}_{V \otimes_{\mathbb{K}} W}=: \tilde{\eta}(V, W): \tilde{F}(V \otimes W) \stackrel{\sim}{\rightarrow} \tilde{F}(V) \otimes \tilde{F}(W)
$$

for all homogeneous objects $V, W \in \operatorname{Vec}_{X}^{\omega}$ of degrees $x, y \in X$, respectively.

The definition of the unit-preserving structure $\eta_{0}$ on $\tilde{F}$ is obvious. It is easy to verify that $\left(\tilde{F}, \tilde{\eta}, \eta_{0}\right)$ is an equivalence of tensor categories.

Next we define isomorphisms $\tilde{\beta}_{g}: F \circ T_{g} \stackrel{\sim}{\rightarrow} T_{f(g)}^{\prime} \circ F$ for $g \in G$ of tensor functors by

$$
\beta_{g}(x) \operatorname{id}_{V}=: \tilde{\beta}_{g}(V):\left(\tilde{F} \circ T_{g}\right)(V) \stackrel{\sim}{\rightarrow}\left(T_{f(g)}^{\prime} \circ \tilde{F}\right)(V)
$$

for all homogeneous objects $V \in \operatorname{Vec}_{X}^{\omega}$ of degree $x \in X$.

It is easy to verify that axioms (i)-(iii) of Definition 2.6 are satisfied. This shows that $\mathscr{C}(\omega, \gamma, \mu, c) \cong \mathscr{C}\left(\omega^{\prime}, \gamma^{\prime}, \mu^{\prime}, c^{\prime}\right)$ as crossed categories.

The converse is clear from the construction above.

Remark 4.3. Proposition 4.2 shows that if the quasiabelian 3-cocycles $(\omega, \gamma, \mu, c)$ and $\left(\omega^{\prime}, \gamma^{\prime}, \mu^{\prime}, c^{\prime}\right)$ are cohomologous (on the same crossed module $(G, X, \partial)$ ), then the corresponding crossed pointed categories $\mathscr{C}(\omega, \gamma, \mu, c)$ and $\mathscr{C}\left(\omega^{\prime}, \gamma^{\prime}, \mu^{\prime}, c^{\prime}\right)$ are equivalent. 
Recall from Section 3 that for any crossed module $\mathscr{X}$ there is a natural action of $\operatorname{Aut}(\mathscr{X})$ on the quasiabelian third cohomology $\mathrm{H}_{\mathrm{qa}}^{3}\left(\mathscr{X}, \mathbb{K}^{\times}\right)$of $\mathscr{X}$.

Theorem 4.4. Crossed pointed categories are classified, up to equivalence, by the orbits of the quasiabelian third cohomology $\mathrm{H}_{\mathrm{qa}}^{3}\left(\mathscr{X}, \mathbb{K}^{\times}\right)$(of a finite crossed module $\mathscr{X})$ under the action of $\operatorname{Aut}(\mathscr{X})$.

Proof. Every crossed pointed category is equivalent to some $\mathscr{C}(\omega, \gamma, \mu, c)$ with underlying (finite) crossed module $\mathscr{X}$. Now apply Proposition 4.2.

\section{Equivariantization of $\mathscr{C}(\omega, \gamma, \mu, c)$}

Throughout this section, let $(\omega, \gamma, \mu, c)$ be a normalized quasiabelian 3-cocycle on a finite crossed module $(G, X, \partial)$. In Section $4 \mathrm{a}$, we associated to $(\omega, \gamma, \mu, c)$ a crossed pointed category $\mathscr{C}(\omega, \gamma, \mu, c)$. Our goal in this section is to apply the equivariantization process to $\mathscr{C}(\omega, \gamma, \mu, c)$ and study the resulting braided fusion category.

5a. Description. Recall that $\mathscr{C}(\omega, \gamma, \mu, c)=\operatorname{Vec}_{X}^{\omega}$ as a fusion category.

Proposition 5.1. An object of the equivariantization category $\mathscr{C}(\omega, \gamma, \mu, c)^{G}$ is an $X$-graded vector space $V$ together with a twisted action $\triangleright$ of $G$ on $V$ that is compatible with the grading in the sense that

$$
\begin{aligned}
g h \triangleright v & =\gamma_{g, h}(x)(g \triangleright(h \triangleright v)), \\
e \triangleright v & =v, \quad \operatorname{degree}(g \triangleright v)={ }^{g} x
\end{aligned}
$$

for all $v \in V$ homogeneous of degree $x \in X$ and $g, h \in G$. Morphisms in the category are linear maps preserving the twisted action and grading. The twisted action of $G$ on the tensor product is given by

$$
g \triangleright(v \otimes w)=\mu_{g}(x, y)(g \triangleright v \otimes g \triangleright w)
$$

for homogeneous $v, w$ of degrees $x, y \in X$, respectively. The associativity constraint on the category is given by

$$
(u \otimes v) \otimes w \mapsto \omega(x, y, z) u \otimes(v \otimes w)
$$

for all homogeneous $u, v, w$ of degrees $x, y, z \in X$. The braiding on the category is given by

$$
v \otimes w \mapsto c(x, y)(\partial(x) \triangleright w \otimes v)
$$

for all homogeneous $v, w$ of degrees $x, y \in X$.

Proof. The action $\triangleright$ corresponds to equivariant structure (1). Equation (4) corresponds to (2). The definition of the tensor product (5) comes from (3) and the definition of the braiding (6) comes from (4). 
Remark 5.2. For a simple special case of the description above, take the quasiabelian 3-cocycle $(\omega, \gamma, \mu, c)$ (on the finite crossed module $(G, X, \partial))$ to be trivial. Then the corresponding equivariantization category $\mathscr{C}(1,1,1,1)^{G}$ admits a simple description in that objects of this category are $G$-equivariant vector bundles on $X$. This braided fusion category was considered in [Bantay 2005]. This category is not nondegenerate in general: By Proposition 5.6 it is nondegenerate if and only if $\partial$ is an isomorphism. In this case, the category is equivalent to $D(G)$-Mod, as a braided fusion category.

Theorem 5.3. Every braided group-theoretical category is equivalent to $\mathscr{b}(\xi)^{G}$ for some normalized quasiabelian 3-cocycle $\xi$ on a finite crossed module $(G, X, \partial)$.

Proof. This follows from [Naidu et al. 2009], where it was shown that every braided group-theoretical category is the equivariantization of a pointed category.

Lemma 5.4. For any $x \in X$, let $\operatorname{Stab}_{G}(x)$ denote the stabilizer of $x$ in $G$, that is, $\operatorname{Stab}_{G}(x)=\left\{\left.g \in G\right|^{g} x=x\right\}$. Define $\phi_{x}: \operatorname{Stab}_{G}(x) \times \operatorname{Stab}_{G}(x) \rightarrow \mathbb{K}^{\times}$by

$$
\phi_{x}(g, h):=\gamma_{g, h}(x) \quad \text { for } g, h \in \operatorname{Stab}_{G}(x) .
$$

Then $\phi_{x}$ is a 2-cocycle on $\operatorname{Stab}_{G}(x)$.

Proof. The condition of Definition 3.4(b) on $\gamma$ means that

$$
\gamma_{h, k}(x) \gamma_{g, h k}(x)=\gamma_{g h, k}(x) \gamma_{g, h}\left({ }^{k} x\right)
$$

for all $g, h, k \in G, x \in X$. Restricting to $\operatorname{Stab}_{G}(x)$ we get the stated assertion.

Let $R$ be a complete set of representatives of orbits of $X$ under the action of $G$.

Proposition 5.5. There is a bijection between the set of isomorphism classes of simple objects of $\mathscr{C}(\omega, \gamma, \mu, c)^{G}$ and the isomorphism classes of the set

$$
\Gamma:=\left\{(a, V) \mid a \in R, V \text { is an irreducible module over } \mathbb{K}_{\phi_{a}}\left[\operatorname{Stab}_{G}(a)\right]\right\},
$$

where $\phi_{a}$ is the 2-cocycle defined in Lemma 5.4.

Proof. Let $\operatorname{Irr}\left(\mathscr{C}(\omega, \gamma, \mu, c)^{G}\right)$ denote the set of simple objects of $\mathscr{C}^{G}$. We will define a map

$$
\Gamma \rightarrow \operatorname{Irr}\left(\mathscr{C}(\omega, \gamma, \mu, c)^{G}\right)
$$

and show that it induces a bijection between the isomorphism classes of the source and target sets. Let $g_{1}, g_{2}, \ldots$ be coset representatives of $\operatorname{Stab}_{G}(a)$ in $G$. Pick any $(a, V) \in \Gamma$. We define the map (8) by

$$
(a, V) \mapsto \tilde{V}=\bigoplus_{g_{i}} V_{s_{i}},
$$


where $V_{g_{i} a}=V$ as a vector space and degree $\left(V_{s_{i} a}\right)={ }^{g_{i}} a$. The twisted action of $G$ on $\tilde{V}$ is given by

$$
h \triangleright v:=\frac{\gamma_{g_{j}, t}(a)}{\gamma_{h, g_{i}}(a)}(t \triangleright v)
$$

for all $v \in \tilde{V}$ homogeneous of degree ${ }^{g_{i}} a$ with $t \in \operatorname{Stab}_{G}(a)$ uniquely determined by the equation $h g_{i}=g_{j} t$. The degree of $h \triangleright v$ is defined to be ${ }^{g_{j}} a$.

To prove that the map (8) defined via (9) and (10) is well defined, we need to show that the action defined in (10) satisfies (4). This amounts to verifying that the scalars

$$
\frac{\gamma_{g_{k}, s t}(a) \gamma_{s, t}(a)}{\gamma_{g h, g_{i}}(a)} \text { and } \frac{\gamma_{g, h}\left({ }^{g_{i}} a\right) \gamma_{g_{j}, t}(a) \gamma_{g_{k}, s}(a)}{\gamma_{h, g_{i}}(a) \gamma_{g, g_{j}}(a)}
$$

are equal for all $g, h \in G$ and $s, t \in \operatorname{Stab}_{G}(a)$ with $h g_{i}=g_{j} t$ and $g g_{j}=g_{k} s$. But this follows from applying the condition (b) on $\gamma$ of Definition 3.4 successively to the quadruples $\left(g, h, g_{i}, a\right),\left(g, g_{j}, t, a\right)$ and $\left(g_{k}, s, t, a\right)$.

We now show that the map (8) induces a bijection between isomorphism classes of the source and target sets. It is clear that the map (8) preserves isomorphic objects. Furthermore, the object in $\operatorname{Irr}\left(\mathscr{C}(\omega, \gamma, \mu, c)^{G}\right)$ corresponding to $(a, V) \in \Gamma$ has FP-dimension equal to $\left|{ }^{G} a\right| \operatorname{dim}_{\mathbb{K}} V$, where ${ }^{G} a$ denotes the orbit containing $a$. The sum of squares of FP-dimensions of isomorphism classes of objects in the image of (8) is

$$
\begin{aligned}
\sum_{a \in R} \sum_{V \in \operatorname{Irr}\left(\mathbb{K}_{\phi_{a}\left[\operatorname{Stab}_{G}(a)\right]}\right)}\left|{ }^{G} a\right|^{2}(\operatorname{dim} V)^{2} & =\sum_{a \in R}\left|{ }^{G} a\right|^{2}\left|\operatorname{Stab}_{G}(a)\right| \\
& =\sum_{a \in R}\left|{ }^{G} a\right||G| \\
& =|G||X|=\operatorname{FPdim}\left(\mathscr{C}(\omega, \gamma, \mu, c)^{G}\right) .
\end{aligned}
$$

5b. Twist and S-matrix. As before, $R$ denotes a complete set of representatives of orbits of $X$ under the action of $G$. By Proposition 5.5, the simple objects of $\mathscr{C}(\omega, \gamma, \mu, c)^{G}$ correspond to pairs $(a, \chi)$, where $a \in R$ and $\chi$ is an irreducible $\phi_{a}$-character of $\operatorname{Stab}_{G}(a)$. Note that $\mathscr{C}(\omega, \gamma, \mu, c)^{G}$ admits a canonical twist $\theta$ with respect to which categorical dimensions coincide with FP-dimensions. The values of $\theta$ on simple objects are given by $\theta_{(a, \chi)}=c(a, a) \chi(\partial(a)) / \operatorname{deg} \chi$. A direct calculation shows that the $S$-matrix $S$ is given by

$$
S_{(a, \chi),\left(b, \chi^{\prime}\right)}=\sum_{\substack{x \in\left({ }^{G} a\right) \\ y \in\left({ }^{G} b\right) \cap C_{X}(x)}} c(x, y) c(y, x) \frac{\gamma_{g, \partial\left(g^{-1} y\right)}(a) \gamma_{h, \partial\left(h^{-1} x\right)}(b)}{\gamma_{\partial(y), g}(a) \gamma_{\partial(x), h}(b)} \chi\left(g^{-1} y\right) \chi^{\prime}\left(h^{h^{-1}} x\right),
$$


where in each summand $g$ and $h$ are defined by ${ }^{g} a=x$ and ${ }^{h} b=y$. Note that the choice of $g$ and $h$ does not affect the sum.

5c. Modularity. As before, $(\omega, \gamma, \mu, c)$ is a normalized quasiabelian 3-cocycle on a finite crossed module $(G, X, \partial)$.

Proposition 5.6. The braided fusion category $\mathscr{C}(\omega, \gamma, \mu, c)^{G}$ is nondegenerate if and only if the homomorphism $\partial$ is surjective and $(\omega, \gamma, \mu, c)$ is nondegenerate in the sense of Definition 3.10.

Proof. This follows immediately by combining Remark 2.5 and Remark 4.1.

Assume $\partial$ is surjective and $(\omega, \gamma, \mu, c)$ is nondegenerate. Then $\mathscr{C}(\omega, \gamma, \mu, c)^{G}$ together with the canonical twist given in Section $5 \mathrm{~b}$ is a modular category, that is, the $S$-matrix described in Section $5 b$ is invertible. In this situation, using the orthogonality relations for projective characters we obtain that the Gauss sum and central charge of $\mathscr{C}(\omega, \gamma, \mu, c)^{G}$ are given respectively by

$$
\begin{aligned}
\tau\left(\mathscr{C}(\omega, \gamma, \mu, c)^{G}\right) & =|G| \sum_{a \in \operatorname{Ker} \partial} c(a, a), \\
\zeta\left(\mathscr{C}(\omega, \gamma, \mu, c)^{G}\right) & =\frac{1}{\sqrt{|\operatorname{Ker} \partial|}} \sum_{a \in \operatorname{Ker} \partial} c(a, a) .
\end{aligned}
$$

Note 5.7. The sum $\sum_{a \in \operatorname{Ker} \partial} c(a, a)$ is the classical Gauss sum for the quadratic form $a \mapsto c(a, a)$ on the abelian group Ker $\partial$.

Remark 5.8. The category $\mathscr{C}(\omega, \gamma, \mu, c)^{G}$ may admit other twists besides the canonical one. In view of Theorem 5.3 and Proposition 5.6, a description of all twists on $\mathscr{b}(\omega, \gamma, \mu, c)^{G}$ will imply a description of all modular group-theoretical categories. The former is easily obtained using Proposition 2.1.

\section{Quasitriangular quasi-Hopf algebra arising from quasiabelian 3-cocycles on crossed modules}

Suppose $(\omega, \gamma, \mu, c)$ is a normalized quasiabelian 3-cocycle on a finite crossed module $(G, X, \partial)$. In the previous section we described the braided fusion category $\mathscr{C}(\omega, \gamma, \mu, c)^{G}$. This category is integral (that is, the FP-dimensions of objects are integers), so there exists a finite-dimensional quasitriangular quasi-Hopf algebra $H$ such that $\mathscr{C}(\omega, \gamma, \mu, c)^{G} \cong H$-Mod, as braided fusion categories; see [Etingof et al. 2005, Theorem 8.33] and [Kassel 1995, Section XV.2]. Our goal in this section is to describe such an $H$.

In what follows we associate to $(\omega, \gamma, \mu, c)$ a finite-dimensional quasitriangular quasi-Hopf algebra $H(\omega, \gamma, \mu, c)$, which may be viewed as a generalization of the twisted Drinfeld double of a finite group. Let $H(\omega, \gamma, \mu, c)$ be a finite-dimensional 
vector space with a basis $\left\{t_{x} g\right\}_{(x, g) \in X \times G}$ indexed by the set $X \times G$. Define a product on $H(\omega, \gamma, \mu, c)$ by

$$
\left(t_{x} g\right)\left(t_{y} h\right):=\delta_{x, h_{y}} \gamma_{g, h}(y)^{-1} t_{y}(g h) .
$$

This product admits a unit

$$
1=\sum_{x \in X} t_{x} e .
$$

Define a coproduct $\Delta: H(\omega, \gamma, \mu, c) \rightarrow H(\omega, \gamma, \mu, c) \otimes H(\omega, \gamma, \mu, c)$ and counit $\varepsilon: H(\omega, \gamma, \mu, c) \rightarrow \mathbb{K}$ by

$$
\begin{aligned}
\Delta\left(t_{x} g\right) & :=\sum_{\substack{a, b \in X \\
a b=x}} \mu_{g}(a, b) t_{a} g \otimes t_{b} g, \\
\varepsilon\left(t_{x} g\right) & :=\delta_{x, e} .
\end{aligned}
$$

Also, set

$$
\begin{aligned}
\Phi & :=\sum_{x, y, z \in X} \omega(x, y, z) t_{x} e \otimes t_{y} e \otimes t_{z} e, \\
R & :=\sum_{x, y \in X} c(x, y) t_{x} e \otimes t_{y} \partial(x), \\
\alpha & :=1, \quad \beta:=\sum_{x \in X} \omega\left(x^{-1}, x, x^{-1}\right) t_{x} e .
\end{aligned}
$$

Finally, define a linear map $S: H(\omega, \gamma, \mu, c) \rightarrow H(\omega, \gamma, \mu, c)$ by

$$
S\left(t_{x} g\right):=\frac{\gamma_{g^{-1}, g}\left(x^{-1}\right)}{\mu_{g}\left(x, x^{-1}\right)} t_{x^{-1}} g^{-1} .
$$

Proposition 6.1. The product unit, coproduct $\Delta$, counit $\varepsilon$, Drinfeld associator $\Phi$ and antiautomorphism $S$ of (11)-(14), (15) and (18) make $H(\omega, \gamma, \mu, c)$ a quasitriangular quasi-Hopf algebra with universal $R$-matrix $R(16)$ in the sense of [Kassel 1995, Definitions 1.1, 2.1, and 5.1].

Proof. The proof is completely similar to the one for the twisted Drinfeld double of a finite group: Associativity of the product is equivalent to the equality

$$
\gamma_{h, k}(x) \gamma_{g, h k}(x)=\gamma_{g h, k}(x) \gamma_{g, h}\left({ }^{k} x\right) \text { for } g, h, k \in G, x \in X,
$$

which holds by axiom (b) in Definition 3.4. Quasicoassociativity of the coproduct is equivalent to the equality

$$
\frac{\mu_{g}(y, z) \mu_{g}(x, y z)}{\mu_{g}(x y, z) \mu_{g}(x, y)}=\frac{\omega\left({ }^{g} x,{ }^{g} y,{ }^{g} z\right)}{\omega(x, y, z)} \quad \text { for } g \in G, x, y, z \in X,
$$


which holds by axiom (c) in Definition 3.4. That the coproduct is a morphism of algebras is equivalent to the equality

$$
\frac{\gamma_{g, h}(x) \gamma_{g, h}(y)}{\gamma_{g, h}(x y)}=\frac{\mu_{g}\left({ }^{h} x,{ }^{h} y\right) \mu_{h}(x, y)}{\mu_{g h}(x, y)} \text { for } g, h \in G \text { and } x, y \in X,
$$

which holds by axiom (d) in Definition 3.4.

We note that the inverse of the $R$-matrix $R$ is

$$
R^{-1}=\sum_{x, y \in X} c\left(x, x^{-1} y x\right)^{-1} \gamma_{\partial(x), \partial\left(x^{-1}\right)}(y)^{-1} t_{x} e \otimes t_{y} \partial\left(x^{-1}\right) .
$$

The $R$-matrix axioms on $R$ hold due to axioms $\left(\mathrm{e}_{1}\right)-\left(\mathrm{e}_{3}\right)$ in Definition 3.4.

Finally, axioms (a)-(d) in Definition 3.4 ensure that $S$ is indeed an antiautomorphism that satisfies the required axioms.

Proposition 6.2. Let $(\omega, \gamma, \mu, c)$ be a normalized quasiabelian 3-cocycle on a finite crossed module $(G, X, \partial)$. The categories $\mathscr{C}(\omega, \gamma, \mu, c){ }^{G}$ (see Section 5) and $H(\omega, \gamma, \mu, c)$-Mod are equivalent as braided fusion categories.

Proof. Let $V$ be a (left) module over $H(\omega, \gamma, \mu, c)$, with action denoted by $\cdot$. Note that $V$ admits an $X$-grading: $V=\bigoplus_{x \in X} V_{x}$, where $V_{x}=\left(t_{x} e\right) \cdot V$. Define a twisted action of $G$ on $V$ by $g \triangleright v:=\left(t_{x} g\right) \cdot v$ for all $v \in V$ homogeneous of degree $x \in X$. Observe that the degree of $g \triangleright v$ is ${ }^{g} x$, since $\left(t_{x} g\right)\left(t_{x} e\right)=\left(t_{g_{x}} e\right)\left(t_{x} g\right)$. The aforementioned action is twisted in that $g h \triangleright v=\gamma_{g, h}(x)(g \triangleright(h \triangleright v))$. Note that the twisted action of $G$ completely determines the action of $H(\omega, \gamma, \mu, c)$ on the module $V$. The associativity constraint on the category $H(\omega, \gamma, \mu, c)$-Mod (which is defined using the Drinfeld associator $\Phi$ of (15)) is given by

$$
(u \otimes v) \otimes w \mapsto \omega(x, y, z) u \otimes(v \otimes w)
$$

for all homogeneous $u, v, w$ of degrees $x, y, z \in X$. The braiding on the category $H(\omega, \gamma, \mu, c)$-Mod (which is defined using the $R$-matrix $R$ of (16)) is given by

$$
v \otimes w \mapsto c(x, y)(\partial(x) \triangleright w \otimes v)
$$

for homogeneous $v, w$ of degrees $x, y \in X$. Now compare with Proposition 5.1.

We next explain the relation between the quasitriangular quasi-Hopf algebras constructed above and the twisted Drinfeld double of a finite group. Let $\omega$ be a normalized 3-cocycle on a finite group $G$.

For all $g, h, x, y \in G$, define

$$
\begin{aligned}
\gamma_{g, h}(x) & :=\frac{\omega(g, h, x) \omega\left(g h x h^{-1} g^{-1}, g, h\right)}{\omega\left(g, h x h^{-1}, h\right)}, \\
\mu_{g}(x, y) & :=\frac{\omega\left(g x g^{-1}, g, y\right)}{\omega\left(g x g^{-1}, g y g^{-1}, g\right) \omega(g, x, y)} .
\end{aligned}
$$


A direct computation establishes the following.

Lemma 6.3. The quadruple $(\omega, \gamma, \mu, 1)$, where $\gamma$ and $\mu$ are respectively defined by (19) and (20), is a quasiabelian 3-cocycle on the crossed module $\left(G, G, \mathrm{id}_{G}\right)$ (where $G$ acts on itself by conjugation) in the sense of Definition 3.4.

Let $(\omega, \gamma, \mu, 1)$ be the quasiabelian 3-cocycle on $\left(G, G, \mathrm{id}_{G}\right)$ constructed in Lemma 6.3. Then, evidently, $H\left(\omega^{-1}, \gamma^{-1}, \mu^{-1}, 1\right) \cong D^{\omega}(G)$ as quasitriangular quasi-Hopf algebras. In particular, $\mathscr{C}\left(\omega^{-1}, \gamma^{-1}, \mu^{-1}, 1\right)^{G} \cong D^{\omega}(G)$-Mod as braided fusion categories.

\section{Acknowledgments}

I thank D. Nikshych and S. Witherspoon for useful discussions.

\section{References}

[Arkhipov and Gaitsgory 2003] S. Arkhipov and D. Gaitsgory, "Another realization of the category of modules over the small quantum group", Adv. Math. 173:1 (2003), 114-143. MR 2004e:17010 Zbl 1025.17004

[Bakalov and Kirillov 2001] B. Bakalov and A. Kirillov, Jr., Lectures on tensor categories and modular functors, University Lecture Series 21, American Mathematical Society, Providence, RI, 2001. MR 2002d:18003 Zbl 0965.18002

[Bantay 2005] P. Bantay, "Characters of crossed modules and premodular categories", preprint, 2005. arXiv math/0512542

[Beliakova and Blanchet 2001] A. Beliakova and C. Blanchet, "Modular categories of types $B, C$ and D", Comment. Math. Helv. 76:3 (2001), 467-500. MR 2003e:57050 Zbl 0996.57003

[Brown 1982] K. S. Brown, Cohomology of groups, Graduate Texts in Mathematics 87, Springer, New York, 1982. MR 83k:20002 Zbl 0584.20036

[Dijkgraaf et al. 1990] R. Dijkgraaf, V. Pasquier, and P. Roche, "Quasi Hopf algebras, group cohomology and orbifold models", Nuclear Phys. B Proc. Suppl. 18B (1990), 60-72. MR 92m:81238 Zbl 0957.81670

[Dijkgraaf et al. 1992] R. Dijkgraaf, V. Pasquier, and P. Roche, "Quasi-Hopf algebras, group cohomology and orbifold models", pp. 75-98 in Integrable systems and quantum groups (Pavia, 1990), edited by P. Binétruy et al., World Sci. Publ., River Edge, NJ, 1992. MR 1178543 Zbl 0925.16024

[Drinfeld et al. 2010] V. Drinfeld, S. Gelaki, D. Nikshych, and V. Ostrik, "On braided fusion categories, I”, Selecta Math. (N.S.) 16:1 (2010), 1-119. MR 2609644 Zbl 05716902

[Eilenberg and Mac Lane 1953] S. Eilenberg and S. Mac Lane, "On the groups of $H(\Pi, n), \mathrm{I}$ ", Ann. of Math. (2) 58 (1953), 55-106. MR 15,54b Zbl 0050.39304

[Eilenberg and Mac Lane 1954] S. Eilenberg and S. Mac Lane, "On the groups $H(\Pi, n)$, II: Methods of computation”, Ann. of Math. (2) 60 (1954), 49-139. MR 16,391a Zbl 0055.41704

[Etingof et al. 2005] P. Etingof, D. Nikshych, and V. Ostrik, "On fusion categories", Ann. of Math.

(2) 162:2 (2005), 581-642. MR 2006m:16051 Zbl 1125.16025

[Gaitsgory 2005] D. Gaitsgory, “The notion of category over an algebraic stack", preprint, 2005. arXiv math.AG/0507192 
[Gelaki and Nikshych 2008] S. Gelaki and D. Nikshych, "Nilpotent fusion categories", Adv. Math. 217:3 (2008), 1053-1071. MR 2009b:18015 Zbl 1168.18004

[Joyal and Street 1993] A. Joyal and R. Street, "Braided tensor categories", Adv. Math. 102:1 (1993), 20-78. MR 94m:18008 Zbl 0817.18007

[Kassel 1995] C. Kassel, Quantum groups, Graduate Texts in Mathematics 155, Springer, New York, 1995. MR 96e:17041 Zbl 0808.17003

[Kirillov 2001a] A. Kirillov, Jr., "Modular categories and orbifold models, II", preprint, 2001. arXiv math/0110221

[Kirillov 2001b] A. Kirillov, Jr., "On $G$-equivariant modular categories”, preprint, 2001. arXiv math/0401119

[Mac Lane 1952] S. MacLane, "Cohomology theory of Abelian groups", pp. 8-14 in Proceedings of the International Congress of Mathematicians (Cambridge, MA, 1950), vol. 2, Amer. Math. Soc., Providence, RI, 1952. MR 13,529d Zbl 0049.01401

[Müger 2003a] M. Müger, "From subfactors to categories and topology, I: Frobenius algebras in and Morita equivalence of tensor categories", J. Pure Appl. Algebra 180:1-2 (2003), 81-157. MR 2004f:18013 Zbl 1033.18002

[Müger 2003b] M. Müger, "On the structure of modular categories", Proc. London Math. Soc. (3) 87:2 (2003), 291-308. MR 2004g:18009 Zbl 1037.18005

[Müger 2004] M. Müger, "Galois extensions of braided tensor categories and braided crossed $G$ categories”, J. Algebra 277:1 (2004), 256-281. MR 2005b:18011

[Müger 2005] M. Müger, "Conformal orbifold theories and braided crossed $G$-categories", Comm. Math. Phys. 260:3 (2005), 727-762. MR 2007b:81228 Zbl 1160.81454

[Naidu et al. 2009] D. Naidu, D. Nikshych, and S. Witherspoon, "Fusion subcategories of representation categories of twisted quantum doubles of finite groups", Int. Math. Res. Not. 2009:22 (2009), 4183-4219. MR 2552301 Zbl 05640426

[Ospel 1999] C. Ospel, Tressages et théories cohomologiques pour les algèbres de Hopf: Application aux invariants des 3-variétés, thesis, Univ. Strasbourg, 1999. MR 2001i:16071

[Ostrik 2003] V. Ostrik, "Module categories, weak Hopf algebras and modular invariants", Transform. Groups 8:2 (2003), 177-206. MR 2004h:18006 Zbl 1044.18004

[Tambara 2001] D. Tambara, "Invariants and semi-direct products for finite group actions on tensor categories", J. Math. Soc. Japan 53:2 (2001), 429-456. MR 2002e:18010 Zbl 0980.18003

[Turaev 2000] V. Turaev, "Homotopy field theory in dimension 3 and crossed group-categories", preprint, 2000. arXiv math/0005291

[Turaev 2008] V. Turaev, "Crossed group-categories", Arab. J. Sci. Eng. Sect. C Theme Issues 33:2 (2008), 483-503. MR 2500054 Zbl 1185.18009

[Whitehead 1949] J. H. C. Whitehead, "Combinatorial homotopy, I", Bull. Amer. Math. Soc. 55 (1949), 213-245. MR 11,48b Zbl 0040.38704

Received June 1, 2009. Revised May 17, 2010.

DEEPAK NAIDU

DEPARTMENT OF MATHEMATICS

TEXAS A\&M UNIVERSITY

College Station, TX 77843-3368

UNITED STATES

dnaidu@math.tamu.edu 


\title{
PACIFIC JOURNAL OF MATHEMATICS
}

\author{
http://www.pjmath.org \\ Founded in 1951 by \\ E. F. Beckenbach (1906-1982) and F. Wolf (1904-1989)
}

\section{EDITORS}

V. S. Varadarajan (Managing Editor)

Department of Mathematics

University of California

Los Angeles, CA 90095-1555

pacific@math.ucla.edu

Vyjayanthi Chari

Department of Mathematics

University of California

Riverside, CA 92521-0135

chari@math.ucr.edu

Robert Finn

Department of Mathematics Stanford University

Stanford, CA 94305-2125

finn@math.stanford.edu

Kefeng Liu

Department of Mathematics

University of California

Los Angeles, CA 90095-1555

liu@math.ucla.edu
Darren Long

Department of Mathematics

University of California

Santa Barbara, CA 93106-3080

long@math.ucsb.edu

Jiang-Hua Lu

Department of Mathematics

The University of Hong Kong

Pokfulam Rd., Hong Kong jhlu@maths.hku.hk

Alexander Merkurjev

Department of Mathematics

University of California

Los Angeles, CA 90095-1555

merkurev@math.ucla.edu
Sorin Popa

Department of Mathematics University of California

Los Angeles, CA 90095-1555 popa@math.ucla.edu

Jie Qing

Department of Mathematics

University of California

Santa Cruz, CA 95064

qing@cats.ucsc.edu

Jonathan Rogawski

Department of Mathematics

University of California

Los Angeles, CA 90095-1555

jonr@math.ucla.edu

\section{PRODUCTION}

pacific@math.berkeley.edu

\begin{abstract}
Silvio Levy, Scientific Editor Matthew Cargo, Senior Production Editor
\end{abstract}
ACADEMIA SINICA, TAIPEI

CALIFORNIA INST. OF TECHNOLOGY

INST. DE MATEMÁTICA PURA E APLICADA

KEIO UNIVERSITY

MATH. SCIENCES RESEARCH INSTITUTE

NEW MEXICO STATE UNIV.

OREGON STATE UNIV.

\section{SUPPORTING INSTITUTIONS}

STANFORD UNIVERSITY
UNIV. OF BRITISH COLUMBIA
UNIV. OF CALIFORNIA, BERKELEY
UNIV. OF CALIFORNIA, DAVIS
UNIV. OF CALIFORNIA, LOS ANGELES
UNIV. OF CALIFORNIA, RIVERSIDE
UNIV. OF CALIFORNIA, SAN DIEGO
UNIV. OF CALIF., SANTA BARBARA

UNIV. OF CALIF., SANTA CRUZ

UNIV. OF MONTANA

UNIV. OF OREGON

UNIV. OF SOUTHERN CALIFORNIA

UNIV. OF UTAH

UNIV. OF WASHINGTON

WASHINGTON STATE UNIVERSITY

These supporting institutions contribute to the cost of publication of this Journal, but they are not owners or publishers and have no responsibility for its contents or policies.

See inside back cover or www.pjmath.org for submission instructions.

The subscription price for 2010 is US \$420/year for the electronic version, and \$485/year for print and electronic.

Subscriptions, requests for back issues from the last three years and changes of subscribers address should be sent to Pacific Journal of Mathematics, P.O. Box 4163, Berkeley, CA 94704-0163, U.S.A. Prior back issues are obtainable from Periodicals Service Company, 11 Main Street, Germantown, NY 12526-5635. The Pacific Journal of Mathematics is indexed by Mathematical Reviews, Zentralblatt MATH, PASCAL CNRS Index, Referativnyi Zhurnal, Current Mathematical Publications and the Science Citation Index.

The Pacific Journal of Mathematics (ISSN 0030-8730) at the University of California, c/o Department of Mathematics, 969 Evans Hall, Berkeley, CA 94720-3840, is published monthly except July and August. Periodical rate postage paid at Berkeley, CA 94704, and additional mailing offices. POSTMASTER: send address changes to Pacific Journal of Mathematics, P.O. Box 4163, Berkeley, CA 94704-0163.

PJM peer review and production are managed by EditFLOW ${ }^{\mathrm{TM}}$ from Mathematical Sciences Publishers.

PUBLISHED BY PACIFIC JOURNAL OF MATHEMATICS

at the University of California, Berkeley 94720-3840

A NON-PROFIT CORPORATION

Typeset in LATEX

Copyright $(\mathrm{C} 2010$ by Pacific Journal of Mathematics 


\section{PACIFIC JOURNAL OF MATHEMATICS}

Volume $247 \quad$ No. $2 \quad$ October 2010

A family of representations of braid groups on surfaces

257

ByUnG HeE AN and Ki HyOUNG Ko

Parametrization of holomorphic Segre-preserving maps

283

R. BLAIR ANGLE

Chern classes on differential $K$-theory

ULRICH BUNKE

Laplacian spectrum for the nilpotent Kac-Moody Lie algebras

DMITRY FUCHS and CONSTANCE WILMARTH

Sigma theory and twisted conjugacy classes

DACIBERG GONÇALVES and DESSISLAVA Hristova Kochloukova

Properties of annular capillary surfaces with equal contact angles

JAMES GORDON and DAVID SIEGEL

Approximating annular capillary surfaces with equal contact angles

JAMES GORDON and DAVID SIEGEL

Harmonic quasiconformal self-mappings and Möbius transformations of the unit ball

David Kalaj and Miodrag S. Mateljević

Klein bottle and toroidal Dehn fillings at distance 5

SANGYOP LEE

Representations of the two-fold central extension of $\mathrm{SL}_{2}\left(\mathbb{Q}_{2}\right)$

HUNG YEAN LOKE and GORDAN SAVIN

Large quantum corrections in mirror symmetry for a 2-dimensional

Lagrangian submanifold with an elliptic umbilic

Giovanni MARELLI

Crossed pointed categories and their equivariantizations

DEEPAK NAIDU

Painlevé analysis of generalized Zakharov equations

Hassan A. ZeDan and Salma M. Al-Tuwairqi 